\title{
'Inside-out', back-to-front: a model for clinical population genetic screening
}

\author{
Darren Shickle, Ian Harvey
}

\begin{abstract}
Developments in DNA technology have resulted in a dramatic increase in the number of genes identified. With the localisation of a gene it is possible to devise procedures suitable for mass carrier screening programmes. Until recently mass carrier screening was only possible for a limited number of disorders, for example, Tay-Sachs disease and haemoglobinopathies. Counselling possible carriers was based on estimations of risk. The momentum towards mass carrier screening is likely to be increased by gene therapy.

Carrier screening for cystic fibrosis alone will have dramatic implications for genetic service provision as 4 to $5 \%$ of the UK population carry the CF gene. The potential for genetic screening of multifactorial diseases, for example, cancers, should also be considered. The existing organisation of genetic services is likely to be inadequate. A new specialty of clinical population genetics is required. A model is proposed of clinical population genetic screening programmes, organised under a 'common umbrella' led by a public health physician, while screening and follow up will remain the responsibility of the appropriate clinician. (f Med Genet 1993;30:580-2)
\end{abstract}

In 1900 , the infant mortality rate for England and Wales was 154 deaths per 1000 live births, with $4 \cdot 5 / 1000$ of the total resulting from genetic disease. By 1980 the infant mortality rate had been reduced to $12 / 1000$. However, the death rate from genetic disorders remained unaltered. $^{1}$ Thus the proportion of infant deaths resulting from genetic disease has increased from 3 to $40 \%$.

Historically, carriers became aware of their genetic status following the diagnosis of an affected child. The couple then had to choose between not trying to conceive further children, adoption, or taking a risk with subsequent pregnancies. It was not possible to inform other family members of their gene status other than an estimation of risk.

For a few disorders, carriers could be detected by haematological or biochemical techniques. For example, subjects with the sickle cell trait possess haemoglobin $S$ and haemoglobin $\mathrm{A}$, and the two forms of haemoglobin will separate on electrophoresis. ${ }^{2}$

Significant advances in DNA technology have revolutionised clinical genetics and dramatically improved options available to geneticists and their patients. Over the last 25 years there has been an almost five fold increase in the number of fully identified genetic disorders. ${ }^{3}$ With the localisation of genes to regions of chromosomes, it became possible to compare the DNA profile of possible carriers with those of affected subjects. However, until the precise gene location was identified and the gene sequenced, there remained an element of doubt about the diagnosis. Even with a more precise determination of gene position and sequence, if there are a number of possible deletions responsible for a condition then DNA from the affected family member may still be needed.

Hence genetic screening has traditionally depended upon the diagnosis of an index case before the identification of carriers. The process is similar to dropping a pebble into water and watching ripples spread out.

Overall fewer than $20 \%$ of pregnancies at risk of important single gene disorders can be predicted on the basis of a positive family history; for multifactorial malformations the figure is only $10 \%$ and for chromosomal disorders under $5 \% .{ }^{1}$ Hence selective testing of pregnancies on the basis of a positive family history, while of benefit to the families concerned, can never have a major impact on the birth frequency of these genetic conditions.

Thus, working outwards from an index case (the 'inwards-outwards' approach) will be an inadequate strategy to deal with a problem of increasing public health importance. For those autosomal recessive conditions where a simple test is available to allow mass carrier screening, a different approach is required. For these programmes, the initial starting point will be screening of subjects with the population level of risk of possessing the abnormal gene in order to identify carriers. This may be followed by screening of partners to identify couples 'at risk', who may be offered prenatal diagnosis when appropriate. In comparison with the traditional model, this could be described as 'outwards-in' (figure).

The skills and organisation required for the 'inwards-out' and 'outwards-in' models may be quite different. Hence, a separate specialty of community genetics has been proposed, ${ }^{4}$ although as yet there is no consensus as to its role and boundaries. The UK Clinical Genetics Society has established a working party in an attempt to produce a consensus definition of community genetics. ${ }^{5}$

The use of the word 'community' may be misleading in that community genetics should not exclude screening performed within an antenatal clinic, simply because it occurs in a hospital. Similarly the definition should not include testing triggered by an index case that 


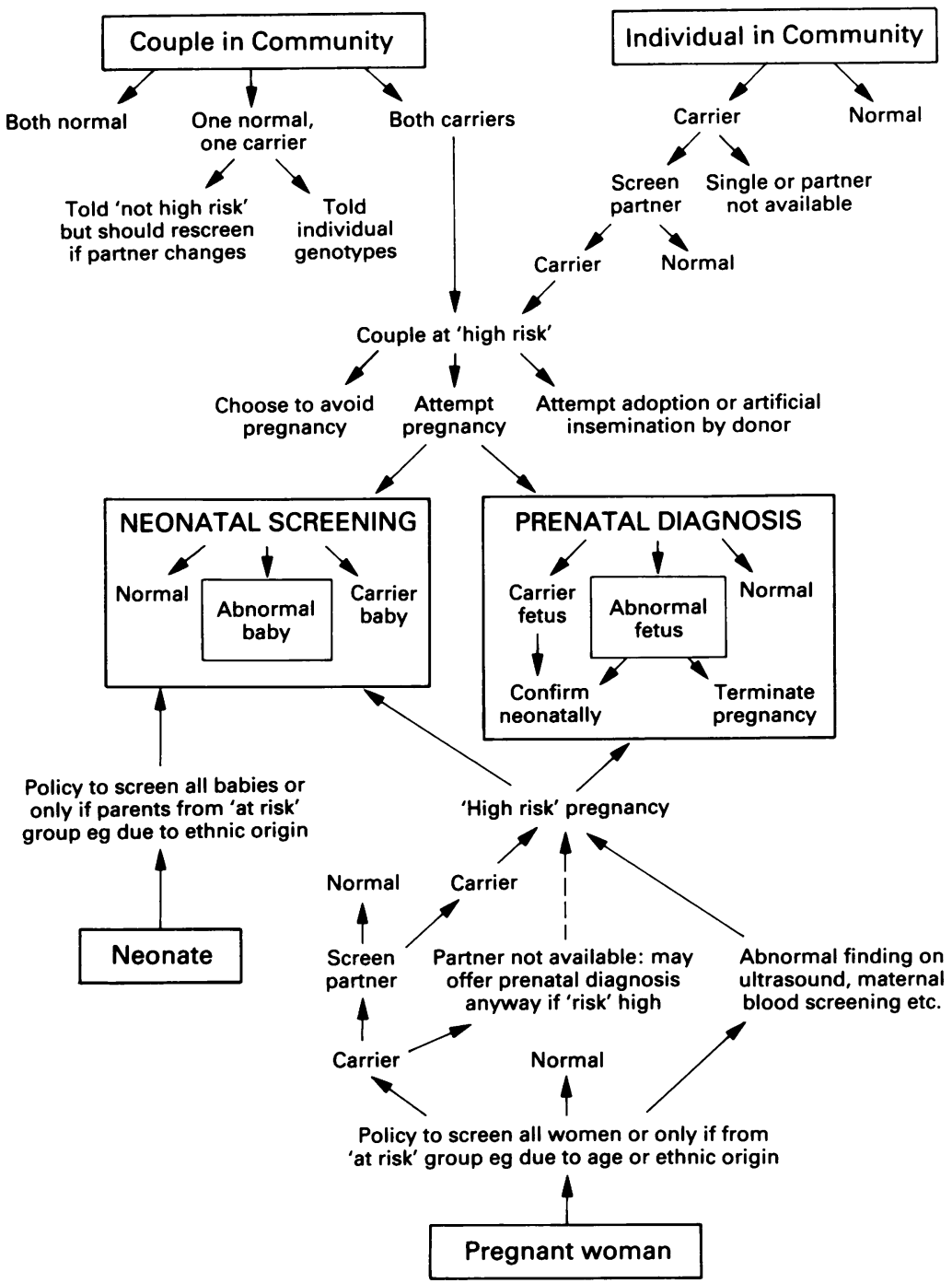

The 'outwards-in' model characterises screening programmes which test individual persons or couples with no previous family history of a genetic abnormality and then work 'inwards' to identify heterozygotes or homozygotes.

happens to be performed in the community rather than in an Institute of Medical Genetics. For example, a 'population screening' programme for fragile $\mathrm{X}^{6}$ involved 'screening' patients with mental handicap to see whether they possess the repeated DNA sequence responsible for the condition, and subsequently offering counselling and testing to family members. However, such a programme would be better described as case ascertainment before using the 'inwards-out' model.

The term 'population genetics' is already used in the context of the study of the origin and dynamics of genetic variation within populations. ${ }^{7}$ Nevertheless, we would advocate the title 'clinical population genetics' in preference to 'community genetics'. This should prevent any confusion over the location of such screening.

Clinical population genetic screening has been performed for many years, for example, neonatal phenylketonuria screening ${ }^{8}$ and prenatal screening for neural tube defects based on maternal serum $\alpha$ fetoprotein. ${ }^{9}$ Carrier screening for haemoglobinopathies ${ }^{2}$ and TaySachs disease $\mathrm{e}^{10}$ are the only mass screening programmes to be consistently successful outside the perinatal period. The lead specialty for each of these examples differs with a large number of departments involved: medical genetics, obstetrics, paediatrics, haematology, medical biochemistry, and general practice. Lack of cooperation and communication have been major obstacles to the provision of multispecialty services. ${ }^{11}$

The increased number of programmes using the 'outwards-in' screening model means that there are advantages to having a 'common umbrella' even if the responsibility for screening and follow up remains with individual specialties. Some genetic screening programmes are relatively small. Thus integration with other programmes would allow exchange of experience and joint appointment of staff. As new screening programmes are approved for implementation, they could be developed alongside the existing services, so facilitating a smoother introduction.

Carrier screening programmes for cystic fibrosis are currently being piloted. ${ }^{12}$ Four to five percent of the population of the UK are carriers of a gene for cystic fibrosis, ${ }^{13}$ that is there are at least 2 million carriers within the UK. In the future, genetic screening may become available for multifactorial diseases such as cancers, ${ }^{14}$ asthma, ${ }^{15}$ and cardiovascular disease. ${ }^{16}$ The momentum for growth of genetic screening is likely to be further increased by gene therapy. ${ }^{17}$ The number of clinical geneticists and specialist counsellors will probably be inadequate to meet future needs. ${ }^{18}$ Instead, the burden is likely to be devolved to general practitioners who may not have the inclination or skills for the task. ${ }^{19}$

The question arises as to which skills are required by a practitioner in clinical population genetics. The wide and increasing range of disorders where screening is feasible means that rather than attempt to train a person with expertise in each, it may be better to identify a person with skills in service development and organisation, who is able to promote interspecialty collaboration. A rapidly developing specialty will also need to include evaluation as a priority, and give close attention to health promotion and ethical considerations. It is for this reason that the involvement of public health medicine has been suggested. ${ }^{420}$ With a public health physician acting as facilitator and coordinator, the individual screening programmes and back up services could be operated by specialists appropriate to each disorder. This model has been used successfully for the breast cancer screening programme in Wales (Breast Test Wales).

The increase in the proportion of infant deaths attributable to genetic disease, improved diagnostic techniques permitting carrier screening, together with gene therapy means that genetics represents a significant area for potential 'health gain' in the future. ${ }^{21}$ However, in view of understandable ethical concerns, ${ }^{22}$ the development of clinical population genetics will require careful consideration. The possibility of screening for 'adult diseases' during childhood will need particular 
attention. ${ }^{23}$ Thus, clinical population genetics should be clearly defined, with aims and objectives to allow evaluation and a formal structure which demands evidence of benefit before investment occurs. The model described of an 'umbrella' structure managed by public health physicians, covering a collection of similar screening programmes using the 'outwards-in' approach, may be a way of ensuring that the promised health gain is secured.

1 Connor JM. Genetic aspects of prenatal diagnosis. $\mathcal{f}$ Inher Metab Dis 1989;12(suppl 1):89-96.

2 World Health Organization. Community control of hereditary anaemias: memorandum from a WHO meeting. Bull WHO 1983;61:63-80.

3 McKusick VA. Mendelian inheritance in man. 9th ed. Baltimore: The Johns Hopkins University Press, 1990.

4 Harper PS. Genetics services in the community. $7 \mathrm{Med}$ Genet 1990;27:473-4.

5 Raeburn JA Delivery of genetic screening services. Birth Defects 1992;28(3):60-8.

6 Turner G, Robinson H, Laing S, et al. Population screening for fragile X. Lancet 1992;339:1210-13.

7 Waldenstrom J. Disease, race, geography and genes. $f$ Intern Med 1990;228:419-24.

8 Medical Research Council Steering Committee for the MRC/DHSS Phenylketonuria Register. Routine neonatal screening for phenylketonuria in the United Kingdom 1964-78. BMf 1981;282:1680-4.

9 Report of UK Collaborative Study on Alpha-fetoprotein in Relation to Neural Tube Defects. Maternal serum alphafetoprotein measurement in antenatal screening for anencephaly and spina bifida in early pregnancy. Lancet 1977;i:1323-32.
10 Kaback M, Nathan T, Greenwald S. Tay-Sachs disease 列 Sachs disease; screening and prevention. New York: Alan R Liss, 1977: 13-36.

11 Wald NJ, Cuckle HS. Open neural-tube defects. In: Wald NJ, ed. Antenatal and neonatal screening. Oxford: Oxford University Press, 1984:66.

12 Watson EK, Mayall E, Chapple J, et al. Screening for carriers of cystic fibrosis through primary health care services. BMY 1991;303:504-7.

13 Working party of Royal College of Physicians on prenatal diagnosis and genetic screening. Prenatal diagnosis and diagnosis and genetic screening. Prenatal diagnosis and genetic screening: community and service implications.

14 Ponder BAJ Molecular genetics of cancer. BMF 1992;304:1234-6.

15 Cookson WOCM, Sharp PA, Faux JA, Hopkin JM. Linkage between immunoglobulin $\mathrm{E}$ responses underlying asthma and rhinitis and chromosome 11q. Lancet 1989;i:1292-5.

16 Berg K. Impact of medical genetics on research and practices in the area of cardiovascular disease. Clin Genet 1989;36:299-312.

17 Report of the Committee on the Ethics of Gene Therapy (Clothier Report). London: HMSO, 1992:Cm 1788.

18 Clinical Genetics Committee of the Royal College of Physicians. Clinical genetic services in 1990 and beyond. London: Royal College of Physicians of London, 1991.

19 Corney RH. Counselling in general practice - does it work? Discussion paper. $₹ R$ Soc Med 1990;83:253-7.

20 Chapple J. Geneticists discover public health - can public health discover genetics? The Public Health Physician, health discover gene

21 Harris R. Medical genetics. BMf 1991;303:977-9.

22 Working Group of the Royal College of Physicians Committee on Ethical Issues in Medicine and Clinical Genetics. Ethical issues in clinic genetics. London: RCP, 1991.

23 Harper PS, Clarke A. Should we test children for 'adult' genetic diseases? Lancet 1991;335:1205-6. 